\title{
Dynamics of the Number of Trades of Financial Securities
}

\author{
Giovanni Bonanno, Fabrizio Lillo and Rosario N. Mantegna \\ Istituto Nazionale per la Fisica della Materia, Unità di Palermo \\ and \\ Dipartimento di Energetica ed Applicazioni di Fisica,Università di Palermo, Viale delle Scienze, I-90128, Palermo, Italia
}

\begin{abstract}
We perform a parallel analysis of the spectral density of (i) the logarithm of price and (ii) the daily number of trades of a set of stocks traded in the New York Stock Exchange. The stocks are selected to be representative of a wide range of stock capitalization. The observed spectral densities show a different power-law behavior. We confirm the $1 / f^{2}$ behavior for the spectral density of the logarithm of stock price whereas we detect a $1 / f$-like behavior for the spectral density of the daily number of trades.
\end{abstract}

89.90.+n

\section{INTRODUCTION}

In the field of econophysics [1] several empirical researches have been performed to investigate the statistical properties of stock price and volatility time series of assets traded in financial markets (for a recent overview see for example [2,3]). Comparably less attention has been devoted to the investigation of statistical properties of the dynamics of the number of trades of a given asset. A similar investigation is relevant for the basic problem of a quantitative assessment of the liquidity of the considered asset. There are two general aspects of liquidity for an asset traded in a market. The first concerns how easily assets can be converted back into cash in a market, whereas the second refers to the general problem of the ability of the market to absorb a given amount of buying and selling of shares of a given security at reasonable price changes. In the present study we consider the statistical properties of trading related to the second aspect of liquidity cited above. Specifically we investigate the temporal dynamics of the number of daily trades of 88 stocks traded in the New York Stock Exchange. The 88 stocks are selected at different values of their capitalization to provide a set of stocks representative of different levels of capitalizations. The interval of capitalization of our set is spanning more than three orders of magnitude in capitalization. With this choice we are able to investigate both heavily traded and less frequently traded stocks. In the present study we focus our attention on the time memory of the dynamics of the number of trades of a set of financial securities. We find that most capitalized stocks are characterized by a dynamics of the number of trades having interesting statistical properties. The time evolution of the number of trades has associated a power spectrum which is well approximated by a $1 / f$ like behavior. We interpret this result as a quantitative evidence that the level of activity of highly capitalized and frequently traded stocks in a financial market does not possess a typical time scale. The same behavior is also qualitatively detected in less capitalized stocks although the value of the exponent $\gamma$ of the power spec- trum $S(f) \propto 1 / f^{\gamma}$ deviates from the value $\gamma=1$ and moves to lower values for stocks with lower capitalization. The modeling of systems with $1 / f$ power spectra is still a theoretical unsolved problem. Hence the level of activity of a given asset in a financial market presents a statistical behavior whose theoretical modeling is certainly challenging. The paper is organized as follows: in the next section we illustrate the analyses performed by discussing the results obtained for one of the most capitalized stocks of the New York Stock Exchange. Section 3 presents the results obtained by investigating the selected set of stocks and in Section 4 we briefly state our conclusions.

\section{SINGLE STOCK ANALYSIS}

We analyze the number of daily trades for a selected set of securities traded in the New York Stock Exchange (NYSE). Our database is the trade and quote (TAQ) database [ 4 f for the three-year period from Jan. 1995 to Dec. 1997. The typical number of daily trades $n(t)$ depends on the stock considered and varies from a few to few thousands units per day. We first analyze the dynamics of the number of daily trades for the General Electrics Co. (GE), which is one of the most capitalized stocks in the NYSE in the investigated period. The time series of the daily number of trades is shown in the inset of Fig. 1. The number of daily trades fluctuates and the time series is non-stationary. In order to test the presence of long range correlation in this time series, we determine its spectral density. The spectral density for the time series of the number of trades of the GE is shown in the upper curve of Fig. 1. For comparison we also show the spectral density of the logarithm of the daily price of the same security. We fit both spectral densities with a power-law function $S(f) \propto 1 / f^{\gamma}$ using a logarithmic binnig in order to not overestimate the high-frequency components. Our best estimation for the exponent $\gamma$ of the spectral density of the logarithm of the price is $\gamma=1.93$. 


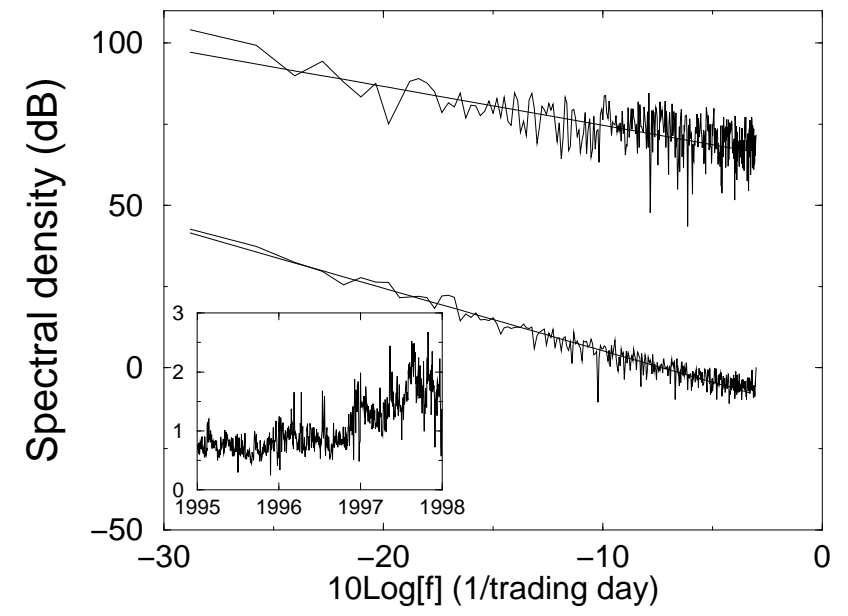

FIG. 1. Log-log plot of the spectral density $S(f)$ of the logarithm of price (bottom curve) and of the number of daily trades (top curve) of the General Electrics Co. (GE). The two spectral densities are well approximated by a power-law behavior $S(f) \propto 1 / f^{\gamma}$ with $\gamma=1.93$ and $\gamma=1.19$, respectively. The two straight lines are our best fit performed as explained in the text. In the inset we show the time evolution of the number of daily trades of GE (in thousands of trades) in the three-year investigated period.

Hence, the spectral density is well approximated by $S(f) \sim 1 / f^{2}$, which is the prediction for the spectral density of a random walk. This form of the spectral density is related to the fact that returns of the price are short time correlated. This result is well-established in financial literature [5.65. We observe a different behavior for the spectral density of the daily number of trades. Our best fit for the exponent $\gamma$ gives $\gamma=1.19$. This value of the exponent is compatible with a $1 / f$ noise [7, 8$]$. The time series of the GE daily trades is non stationary and shows a clear trend. We check the effect of these features on the fitting of the spectral density by investigating surrogate data obtained removing the trend by evaluating a running average of the original time series computed using a window of length $L$. With this procedure, we obtain a detrended time series by dividing the original number of trades at a given day $t$ by the average of the number of trades in a period of length $L$ centered in $t$. We take the values of $L$ equal to 11,21, 41 and 81 trading days. The new time series do not present a global trend. In Fig. 2 we scomponents lower than $1 / L$ are affected by the running average procedure. We perform a power law fit in the interval of frequency higher than $1 / L$ and our best fit for the exponent $\gamma$ gives $\gamma=1.09,0.85,0.85,0.91$ for $L=11,21,41,81$, respectively. The estimation of the exponent is weakly affected by the running averaging procedure. These results for the spectral density in the original and detrended time series indicate that the number of trades is intrinsically long-range correlated.

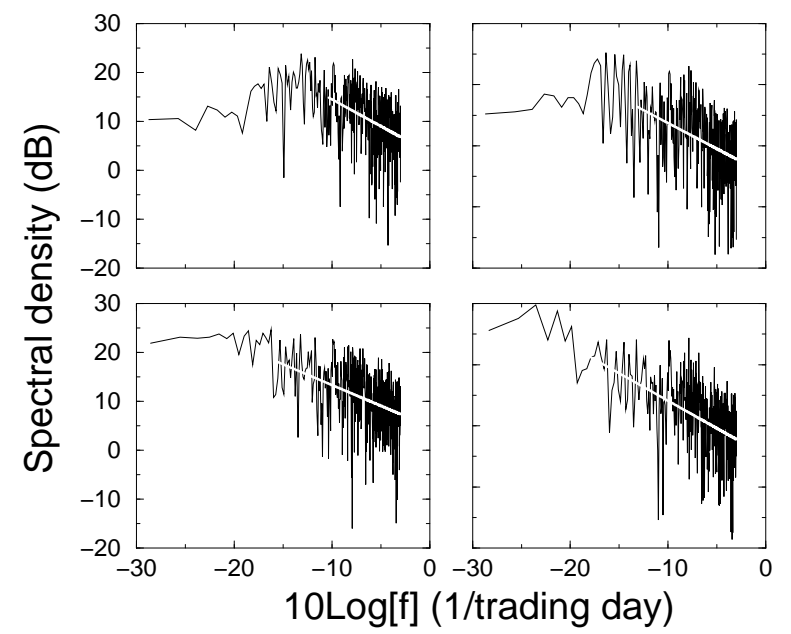

FIG. 2. Spectral densities of the number of daily trades obtained by detrending the original time series. The detrending is obtained by dividing the time series by the running average in a time window of length $L$. In the four panels we show the spectral densities obtained by detrending the original time series with averaging windows of length $L=11$ (top left), $L=21$ (top right), $L=41$ (bottom left) and $L=81$ (bottom right) trading days. In the regions not affected by the detrending procedure $(f>1 / L)$ a $1 / f$-like behavior is always observed (solid line).

\section{COMPARISON BETWEEN STOCKS}

In this section we study the general validity of the results illustrated in the previous section by studying a specific stock. In particular we determine the spectral density of the logarithm of the price and of the number of daily trades for a set of 88 selected stocks traded in the NYSE. We select the stocks randomly in a wide range of capitalization. The capitalization of our set ranges from $32 \cdot 10^{6}$ USD, which is the capitalization of the Sun Energy (SLP), to $80 \cdot 10^{9}$ USD for the GE. We determine the spectral density for the $\ln S_{i}(t)$ and of the $n_{i}(t)$, where $S_{i}(t)$ and $n_{i}(t)$ are the closure price and the number of trades of company $i$ at day $t$, respectively. The subscript $i=1, \ldots, 88$ labels the company. For each spectral density we perform a power-law fit $S(f) \propto 1 / f^{\gamma}$. We check the robustness of our fittings by determining their correlation coefficient and we find absolute values ranging from 0.87 to 0.99 (average value 0.97 ) for the logarithm of the price and from 0.56 to 0.96 (average value 0.86 ) for the number of trades. In Fig. 3 we show the two exponents as a function of the capitalization of the investigated security. The large majority of values of the exponent $\gamma$ of the spectral densities of the logarithm of the price is close to the value $\gamma=2$. On the other hand the large majority of values of the exponent $\gamma$ of the spectral densities of the number of daily trades is close to the value $\gamma=1$, in particular for the most capitalized stocks. Moreover, the exponent $\gamma$ increases towards the value $\gamma=1$ as far as the capitalization increases. When we consider less cap- 
italized stocks the typical number of daily trades can be very small, of the order of 10 or less. This fact leads to a problem of high digitalization noise of the time series,

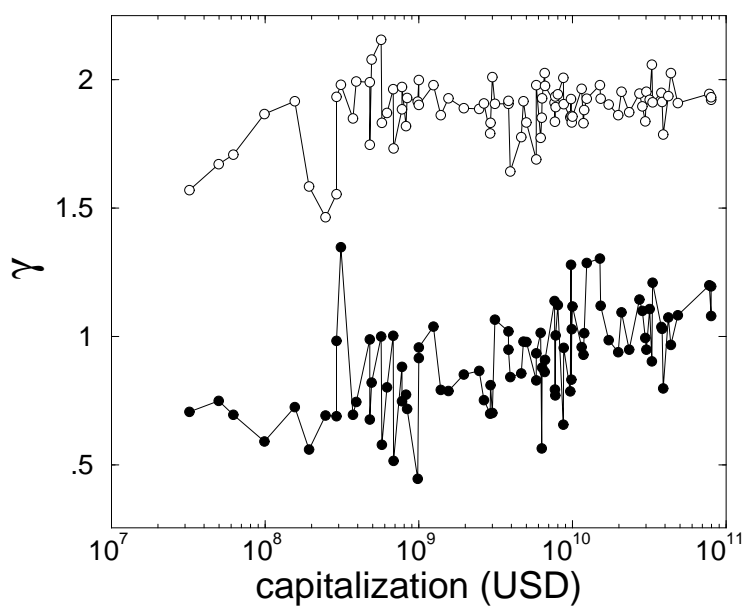

FIG. 3. Plot of the exponent $\gamma$ of the power-law fit $S(f) \propto 1 / f^{\gamma}$ of the spectral density of the number of trades (filled circle) and of the logarithm of the price (empty circle) as a function of the capitalization of the investigated company. The exponent $\gamma$ for the logarithm of the price is almost constant for the majority of stocks and clusters in the neighborhood of $\gamma=2$. On the contrary, for the number of trades, the exponent $\gamma$ slowly increases by increasing the capitalization and clusters in the neighborhood of $\gamma=1$ for the most capitalized stocks.

because the number of trades is an integer and the quantization noise is relevant. As a consequence the statistical analysis on these time series may present a non negligible white noise contribution. This fact may be one reason for the observed decreases of the value of the exponent $\gamma$ in less capitalized stocks.

\section{CONCLUSION}

The statistical properties of (i) the logarithm of the price and (ii) the number of daily trades of a security traded in a financial market are quite different. Specifically, we confirm that the time series of the logarithm of the price are characterized by a $1 / f^{2}$ spectral density, whereas we observe that the time series of the daily number of trades show a $1 / f$-like spectral density. The $1 / f^{2}$ behavior observed in the time evolution of the logarithm of the price reflects the short time temporal memory of price returns. This kind of short-range time memory is needed to ensure absence of arbitrage opportunities in the market. On the other hand the $1 / f$-like behavior observed in the daily number of trades manifests the absence of a typical scale in the time memory of this variable. In other words the activity of trading is not constant in time even for most capitalized stocks and its modeling needs to take into account phenomena occurring at a variety of time scales. Realistic models of trading activities in financial markets should take into account this feature empirically observed.

\section{ACKNOWLEDGEMENTS}

The authors thank INFM and MURST for financial support. This work is part of the FRA-INFM project 'Volatility in financial markets'. G. Bonanno and F. Lillo acknowledge FSE-INFM for their fellowships.

[1] R. N. Mantegna and H. E. Stanley, An Introduction to Econophysics: Correlations and Complexity in Finance, (Cambridge Univ. Press, in press).

[2] R. N. Mantegna, ed., Proceedings of the International Workshop on Econophysics and Statistical Finance, Physica A 269, Issue 1 (1999).

[3] J. P. Bouchaud, ed., Proceedings of the International Conference on Application of Physics in Financial Analysis, International Journal of Theoretical and Applied Finance in press (2000).

[4] The Trade and Quote Database, (New York Stock Exchange, New York), http://www.nyse.com/ marketinfo/taqdatabase.html.

[5] P. H. Cootner, ed., The Random Character of Stock Market Prices, (MIT Press, 1964).

[6] P. A. Samuelson, Industrial Management Rev. 6, 41 (1965).

[7] W. H. Press, Comments Astrophys. 7, 103 (1978).

[8] B. B. Mandelbrot, Multifractals and 1/F Noise: Wild SelfAffinity in Physics (1963-1976), (Springer Verlag, 1999). 